\title{
LA DELIBERACIÓN EN LA TOMA DE DECISIONES BIOÉTICO-CLÍNICAS SEGÚN DIEGO GRACIA
}

Mario Orlando Parra Pineda ${ }^{1}$

\section{Resumen}

Introducción. El objetivo de este artículo de reflexión es estudiar el método deliberativo para la toma de decisiones en bioética clínica expuesto por Diego Gracia, una de las voces más reconocidas en el campo de la bioética española. En este artículo se analizan los siguientes aspectos de su propuesta: la fundamentación bioética, la deliberación, el proyecto humano, el acto moral, el método de toma de decisiones, las dificultades para la deliberación y las críticas al método. Métodos. la fundamentación epistemológica de esta investigación es interpretativa, el enfoque cualitativo, el diseño analítico y la técnica de investigación utilizada es el análisis documental. Las categorías de análisis de este estudio son: fundamentación bioética, deliberación, proyecto humano, acto moral, método de toma de decisiones, dificultades para la deliberación y críticas al método. Resultados. los aportes más importantes de esta propuesta son: la valoración de la historia clínica como el elemento básico para el estudio y el análisis del caso clínico; el reconocimiento de la deliberación prudente como el eje fundamental para el estudio de las situaciones problemas; el estudio que se hace de las dificultades intrínsecas y extrínsecas que pueden dificultar o entorpecer el proceso deliberativo; y que la metodología propuesta es clara y sencilla para la identificación y elección de los problemas morales a estudio y de los cursos de acción para su resolución. Discusión. Es de reconocer la obra de Diego Gracia como una de las contribuciones más importantes realizadas al estudio de los métodos de toma de decisiones en bioética clínica en lengua española, la cual se constituye como una herramienta útil en la construcción y enriquecimiento del conocimiento en este campo para los profesionales de la salud y los comités bioéticos clínicos.

${ }^{1}$ Estudiante del Doctorado en Bioética. Facultad de Educación y Humanidades. Universidad Militar Nueva Granada. Sede Cajicá. Colombia. Profesor Asociado del Departamento de Obstetricia y Ginecología. Facultad de Medicina. Universidad Nacional de Colombia. Sede Bogotá. Colombia. Correspondencia: Mario Parra Pineda. Facultad de Medicina. Universidad Nacional de Colombia. Carrera 30 No. 45 - 03. Oficina 136. Teléfono: +571 3165000, extensión: 15167. Bogotá, D.C., Colombia.Correo electrónico: moparrap@unal.edu.co 


\title{
THE DELIBERATION IN BIOETHICAL CLINICAL DECISIONS MAKING ACCORDING TO DIEGO GRACIA
}

Mario Orlando Parra Pineda ${ }^{1}$

\begin{abstract}
Introduction. The objective of this reflection article is to study the deliberative method for decision making in clinical bioethics presented by Diego Gracia, one of the most recognized voices in the field of Spanish bioethics. In this article the following aspects of his proposal will be analyzed: the bioethical fundamentals, deliberation, human project, moral act, the decision-making method, the difficulties for the deliberation and criticism of this method. Methods. The epistemological foundation of this research is interpretive, the qualitative approach, the analytical design and the research technique used in the documentary analysis. The categories of analysis of this study were: bioethical foundation, deliberation, human project, moral act, method of decision making, difficulties for deliberation and criticism of this method. Results. The most important contributions of this proposal are: a) the evaluation of the clinical history as the main subject for the clinical case study and analysis; b) the recognition of prudent deliberation as the main axis for the study of problematic situations; $c$ ) the study that is made of the intrinsic and extrinsic difficulties that can make difficult or obstruct the deliberative process; and d) the proposed methodology is clear and simple to identify and select moral problems to study and courses of action for their resolution. Discussion. It is to recognize the work of Diego Gracia as one of the most important contributions made to the study of decision-making methods in clinical bioethics in Spanish, which is a useful tool in the construction and enrichment of knowledge in this field for health professionals and clinical bioethics committees.
\end{abstract}




\title{
A DELIBERAÇÃO EM TOMADA DE DECISÃO EM BIOÉTICA CLÍNICA DE ACORDO COM DIEGO GRACIA
}

\author{
Mario Orlando Parra Pineda ${ }^{1}$
}

\section{Resumo}

Introdução. O objetivo deste artigo de reflexão é estudar o método deliberativo de tomada de decisão em bioética clínica exposto por Diego Gracia, uma das vozes mais reconhecidas no campo da bioética espanhola. Neste artigo serão analisados os seguintes aspectos da proposta acima referida: fundamentos bioéticos, deliberação, projeto humano, o ato moral, o método de tomada de decisão, dificuldades para a deliberação e crítica ao método. Métodos. O fundamento epistemológico desta pesquisa é interpretativo, a abordagem é qualitativa, o desenho é analítico e a técnica de pesquisa utilizada na análise é documental. As categorias de análise deste estudo foram: fundamentação bioética, deliberação, projeto humano, ato moral, método de tomada de decisão, dificuldades de deliberação e crítica a esse método. Resultados. As contribuições mais importantes desta proposta são: a) a avaliação da história clínica como elemento básico para o estudo e análise do caso clínico; b) o reconhecimento da deliberação prudente como eixo fundamental para o estudo de situações-problema; c) o estudo que é feito das dificuldades intrínsecas e extrínsecas que podem dificultar ou obstruir o processo deliberativo; d) a metodologia proposta é clara e simples para a identificação e eleição dos problemas morais a serem estudados e os cursos de ação para sua resolução. Discussão. O trabalho de Diego Gracia deve ser reconhecido como uma das contribuições mais importantes ao estudo dos métodos de tomada de decisão em bioética clínica em língua espanhola e constitui uma ferramenta útil na construção e enriquecimento de conhecimento neste campo para profissionais de saúde e comitês de bioética clínica. 


\section{Introducción}

El profesor Diego Gracia Guillén es una de las personalidades de mayor reconocimiento en el campo de la bioética española. En su obra, se pone de manifiesto un gran interés por el estudio de los problemas de salud, especialmente en relación con los métodos para la toma de decisiones en bioética clínica(1-3). El presente escrito es un artículo de reflexión, por ser el resultado de una investigación analítica en el campo de la bioética clínica, cuyo objetivo es el de analizar su segunda propuesta para el estudio de los problemas bioéticos clínicos basada en la deliberación(4); dado que se considera una herramienta valiosa para su aplicación por parte de los comités de bioética clínica.

La bioética es una disciplina de, relativamente, reciente aparición (en la década de los setenta), que tiene su origen en los trabajos del profesor Van Rensselaer Potter quien la define como "el conocimiento de cómo usar el conocimiento para la superviviencia humana y para mejorar la condición humana" (5,p.32), y que en el área clínica tiene por propósito “...el proceso de contrastación de los hechos biológicos con los valores humanos, a fin de globalizar los juicios sobre las situaciones y de esta forma mejorar la toma de decisiones, incrementado su corrección y calidad" (6, p.30). En este contexto, los métodos de toma de decisiones en bioética clínica son instrumentos propuestos para el estudio sistemático y ordenado de los problemas morales que se presentan en el contexto de la relación clínica, en procura de la mejor solución desde el punto de vista humano, ético y científico (6). Asimismo, son de gran utilidad para los profesionales de la salud en su ejercicio profesional y los comités de bioética clínica, órganos colegiados consultivos que tienen por propósito el "procurar resolver los conflictos éticos que plantea la asistencia hospitalaria" (7,p.19).

\section{Metodología de la investigación}

La fundamentación epistemológica de esta investigación es interpretativa(8); su enfoque cualitativo $(8,9)$; y su diseño analítico. Se considera como una estrategia investigativa con fines no solamente descriptivos sino también explicativos y tiene por propósito la interpretación y comprensión de la propuesta deliberativa de Gracia(9). La técnica de investigación utilizada fue el análisis documental, a partir de una revisión bibliográfica previa que tuvo por objetivo la condensación de la información pertinente respecto a este tema. Para su estudio se han propuesto las siguientes categorías de análisis, que surgieron del mismo estudio realizado a los textos: fundamentación bioética, deliberación, proyecto humano, acto moral, método de toma de decisiones, dificultades para la deliberación y críticas a este método(10).

\section{Resultados}

\section{La fundamentación bioética}

El método propuesto se encuentra cimentado en el concepto del valor desde la perspectiva del constructivismo axiológico, la cual se sustenta en el trabajo 
de Xavier Zubiri y en la ética formal de bienes. En este se reconoce, por una parte, que el conocimiento inicial de la realidad se realiza de manera simultánea, intelectual y emocional, "dos momentos de un solo acto de aprehensión sentiente de lo real"(11, p.12), a través de lo que él ha denominado la inteligencia sentiente; y por otra parte, la posibilidad de la realidad de ser conocida formalmente, la cual, por su misma naturaleza, se constituye en un bien, en algo bueno capaz de ser conocido; en donde su valoración primera se hace mediante un proceso de preferencia, por medio del cual una realidad o bien es seleccionada o no en relación a otra.(11, 7).

A partir de este sistema de referencia inicial es posible en el campo moral construir y justificar racionalmente de manera deliberativa una escala de valores objetiva, universal y jerárquica; y posteriormente, una moral normativa propiamente dicha. De acuerdo con Gracia, "la universalidad de nuestras proposiciones no tiene por qué buscarse en el orden meramente subjetivo. Podemos universalizar las categorías morales de forma intersubjetiva, a través de un diálogo en el que participen actual o virtualmente todos los seres humanos" (3, p.609).

\section{La deliberación}

La deliberación (vocablo latino, deliberatio; y del griego, $\sigma \dot{\sigma \kappa \varepsilon} \psi \eta$ ) ha sido definida por la Real Academia Española como la acción y efecto de deliberar, entendiéndose por este, el "considerar atenta y detenidamente el pro y contra de los motivos de una decisión, antes de adoptarla, y la razón o sinrazón de los votos antes de emitirlos // resolver con premeditación"(12). En esencia, este es un proceso para la toma de decisiones a partir del "análisis detenido de las circunstancias y consecuencias que concurren a una situación concreta, y por tanto también en la identificación de los cursos de acción posibles y de entre todos ellos, del curso óptimo"(13, p.29). Es una habilidad que se reconoce como la base para la toma de decisiones en la vida cotidiana; "todos los seres humanos deliberamos, y deliberamos continuamente. No podemos vivir sin deliberar. Y ello porque se trata de una necesidad biológica"(14, p.109), y en los campos especializados del saber, como la medicina, el derecho, la política y la ética, desde la antigüedad $(15,16)$. Esto ha llevado a Gracia a denominarla como el "método de la razón práctica" (17, p.32) y a considerarla como una característica esencial de la naturaleza humana que lo define como un animal deliberans(14, p.113).

Esta habilidad permite el análisis de las situaciones complejas y la búsqueda de la mejor solución, a partir del reconocimiento de la naturaleza contingente y probabilística del conocimiento humano. A este respecto, el empirismo inglés (siglo XVII) ya reconocía “...que todo saber empírico es imperfecto, y que esa imperfección sube de grado cuando intenta formularse proposiciones universales, como hace la ciencia... No hay conocimiento empírico absolutamente verdadero"(18, p.25).

De ahí que las decisiones que se tomen siempre tengan algún grado de incertidumbre y probabilidad, alejadas de la certeza y verdad absoluta propias de la lógica y las matemáticas (19), y que este 
sea un proceso abierto que permite $y$ promueve la participación libre, prudente y racional de todos los interesados, a partir de sus propias realidades, en pro de la construcción de una solución de la mayor calidad y corrección $(17,20)$. Algunas de sus características más importantes son (14):

- Es un proceso mental común de los seres humanos para la toma de decisiones de manera racional, que tiene por finalidad orientar su acción en el mundo.

- Tiene por objeto hechos concretos, una situación-problema sobre la cual se tiene la capacidad de modificar o cambiar, "puesto que no se delibera sobre lo que no depende de nosotros" (19, p.61).

- La toma de decisiones se hace de manera racional y argumentada, en condiciones de incertidumbre y teniendo en cuenta las circunstancias propias de la situación problema y las consecuencias previsibles del acto.

- Generalmente, al final del proceso se logra tomar una decisión, que se considera como la de mayor calidad y corrección $(14,20)$. Sin embargo, hay situaciones en que es posible considerar más de una solución posible; en otros casos, los menos, no es posible llegar a una. "Y cuando esto sucede, lo que habrá que hacer es dejar una cierta libertad a las diferentes concepciones, o a las diferentes soluciones" (19, p.69).

- Puede ser de carácter individual o grupal. En sociedad, la toma de decisiones se ve enriquecida cuando esta se construye de una manera consensuada a través del diálogo y la fuerza de la argumentación, a partir de las diferentes perspectivas de mundo.

- A nivel individual, propende a dar las bases para ser mejores seres humanos, profesionales y ciudadanos; y en el ámbito social, a la búsqueda de la mejor solución posible para todos los representados, presentes y futuros. De ahí la importancia de educar en este proceso a los seres humanos a largo de todo el ciclo formativo (21).

\section{El proyecto humano}

De acuerdo con Gracia, la deliberación ha permitido a los seres humanos planear sus acciones de una manera efectiva sobre su entorno, con el fin de adecuarlo y transformarlo a su favor a lo largo de la historia, humanizando el medio y, por ende, creando cultura. Se ha logrado a través de acciones orientadas mediante proyectos, en los que es posible identificar tres etapas interrelacionadas, que caracteriza así $(14,22)$ :

Y el proyecto humano consta siempre de tres fases, una cognitiva, que identifica los hechos relevantes para el proyecto que hemos concebido; otra emocional, que valora el proyecto de transformación de los hechos; y una tercera práctica, que pone en obra el proyecto, que lo realiza, lo lleva a cabo haciéndolo realidad (14, p.110).

\section{El acto moral}

De igual forma en que el ser humano proyecta y realiza sus acciones en la vida 
cotidiana también lo hace en el ámbito moral, en favor de la autorrealización de sí mismo y los demás, y se reconoce éste como el objetivo primordial de la moral(23). En el acto moral se identifican las mismas tres etapas del proyecto humano: hechos, valores y acciones o deberes. Algunas de sus características más importantes son:

- Los hechos: el acto humano se inicia con la interacción del hombre con su medio y su necesidad de dar respuesta a sus necesidades. Este proceso parte de la interacción del ser humano con la realidad, en donde esta se presenta como hechos independientes que permiten ser conocidos (24).

- Los valores: posteriormente a la aprehensión de la realidad, es posible hacer una estimación del valor del objeto de estudio y realizar juicios al respecto que permitan establecer criterios de preferencia entre diferentes hechos o acciones (23).

- Los deberes: a partir de esta valoración de los hechos es posible determinar los mejores cursos de acción a realizar, que en el campo moral adquieren el estatus de deberes; "Ahora bien, lo valores tienen una característica no señalada pero esencial ahora: piden su realización. Y esto sí que es lo propio de la ética: realizar valores" (24, p.14).

\section{El método de toma de decisiones deliberativo}

Gracia estructura su método de toma de decisiones teniendo en cuenta las mismas etapas del proyecto humano y el acto moral. Se presenta una síntesis del método, basada en su artículo "Teoría y práctica de la deliberación moral” (14, p.101-154):

- La deliberación sobre los hechos: hace referencia a la necesidad del conocimiento del caso de la manera más completa posible, desde el punto de vista científico y humano, "reduciendo la incertidumbre sobre ellos a límites razonables y prudentes" (14, p.122), como base para la toma de decisiones. De igual forma, como en su primera propuesta metodológica, se enfatiza en la necesidad de contar con una historia clínica completa, que permita el análisis a partir de hechos ciertos y suficientes (25). En esta etapa se identifican dos momentos: a) la presentación del caso; y b) la deliberación acerca de este, en la que se realizan las preguntas necesarias para su comprensión.

- La deliberación sobre los valores: A partir del conocimiento de los hechos se procede, en primer lugar, a la identificación de los problemas morales. De acuerdo con la experiencia de este autor, no deben ser menores a siete, teniendo en cuenta que un "problema moral puede considerarse aquí todo aquello que es un problema moral para alguien" (24, p.28). Se caracteriza por evidenciar un conflicto de valores positivos, "parece evidente que un conflicto de valores no es más que una colisión de dos valores, no porque esos dos valores sean siempre opuestos, sino porque en una situación concreta resulta imposible realizar uno 
sin lesionar al mismo tiempo el otro" (24, p.27). En segundo lugar, se realiza la identificación del problema moral a estudiar. A partir del listado de problemas propuestos se procede a seleccionar el problema moral más importante para su estudio; en caso de que se identifique más de un problema relevante, estos se analizarán de manera sucesiva e independiente en una o más sesiones. En esta selección es deseable que participen la o las personas que traen el caso a evaluación. En tercer lugar, se hace la identificación de los valores en conflicto que se encuentran en juego.

- La deliberación sobre los deberes: en este aparte, se estudian los cursos de acción posibles que busquen dar solución al problema y a los valores en conflicto. Los momentos recomendados para ello son:

a) la identificación de los cursos de acción: inicialmente, se identifican los cursos de acción extremos "un curso extremo es aquel que, optando por un valor..., lesionamos completamente el otro" (24, p. 29); y posteriormente, los intermedios. Respecto a esto, Gracia menciona:

El curso óptimo se halla siempre entre los intermedios. Es fundamental no perder de vista que solo estamos legitimados para elegir un curso extremo cuando han fracasado todos los cursos intermedios... (14, p.147148).

En este proceso se recomienda que los cursos de acción identificados no sean menores a cinco (13). b) la elección de curso de acción óptimo: a partir de los cursos de acción identificados, se procede a seleccionar el o los cursos de acción que mejor den solución a los valores en conflictos, de acuerdo con las circunstancias y las consecuencias previsibles del caso $(18,24)$.

Las pruebas de consistencia de la decisión: en esta etapa se somete el curso de acción seleccionado a las siguientes preguntas, "para asegurar que la decisión que se va a tomar es prudente y responsable" (24, p.31):

Estabilidad temporal. ¿Se tomaría la misma decisión transcurrido un tiempo? Una forma de garantizar este proceso es disponiendo del tiempo suficiente para ello, lo que de alguna manera evita que sus conclusiones sean producto de las emociones o de otras circunstancias que las invaliden.

c) Publicidad. ¿Se estaría dispuesto a defenderlo públicamente? Esto permite asegurar que la decisión que se tome sería la que se debería recomendar en todos los casos que le sean similares, lo que de alguna forma trata de garantizar el principio de universalización en la toma de decisiones.

d) Legalidad. ¿Es legal el curso recomendado? Se contrasta la decisión con el marco legal para garantizar su corrección $(1,14,18)$.

- La decisión final: el comité de bioética recomendará el curso de acción que mejor haya cumplido con este 
proceso, pero su cumplimiento será responsabilidad de la institución de salud, el profesional de salud, el paciente y/o familiares. La conclusión elaborada tiene el carácter de recomendación, por lo que no es de obligatorio cumplimiento, aunque por su naturaleza deliberativa y colegiada siempre es aconsejable su acatamiento (1).

\section{Dificultades de la deliberación}

Gracia reconoce algunas dificultades intrínsecas y extrínsecas que pueden dificultar o entorpecer el proceso de la deliberación. Deben ser reconocidas de manera oportuna, con el fin de evitar que se constituyan en un obstáculo en esta tarea. $\mathrm{Al}$ respecto, se mencionan las siguientes (14):

- La realidad por naturaleza es compleja y dinámica, de ahí que las decisiones que se tomen sobre esta se caracterizan por la incertidumbre. Por tanto, la toma de decisiones se basa en la probabilidad y no en la certeza, la cual puede ser susceptible de verificación en el tiempo.

- El conocimiento de la realidad se encuentra en constante evolución y perfeccionamiento. El reconocimiento de esta realidad obliga a los seres humanos a reevaluar sus propias creencias con el fin de poder dar respuesta a los retos que esta misma plantea de forma permanente.

- La comprensión de la realidad moral requiere de seres humanos autónomos, libres e iguales, con la capacidad de entenderla y transformarla, es decir, sujetos que pasan de aceptar y obedecer normas y reglas morales externas (heteronomía), a hacerlas suyas (autonomía); a ser sus propios dueños.

- Las convicciones de los seres humanos frente al mundo son su acerbo para su comprensión y supervivencia. La habilidad para poder cambiar estas convicciones implica para el individuo el tener la capacidad para tolerar el cambio y el manejo de la incertidumbre. La incapacidad para hacerlo genera temor a lo desconocido y el resquebrajamiento de la seguridad previa, lo que ocasiona en la persona respuestas inconscientes de defensa. Así mismo, esta incapacidad de manejar la incertidumbre no permite reconocer al otro como un interlocutor válido $y$, por ende, la comunicación se cierra antes de comenzar el proceso deliberativo.

- La deliberación puede verse dificultada por la participación de sus miembros a partir de posiciones ideológicas reduccionistas y de carácter absoluto que no permiten su participación activa en este proceso. Las más importantes identificadas por el autor son:

a) el reduccionismo técnico, que reduce el análisis de la realidad a los hechos, a lo objetivable y cuantificable, dejando de lado otros aspectos, como los valores, elementos fundamentales para una valoración integral de la situación problema en las ciencias sociales.

b) el reduccionismo religioso, que circunscribe el análisis de los problemas morales a los valores o normas determinados previamente por una 
religión, por lo que se reduce el estudio del caso a la dimensión deontológica. Sin embargo, es de resaltar la importancia de los aportes que las religiones han hecho a la vida moral, los cuales deben ser tenidos en cuenta en las discusiones de situaciones problémicas para la búsqueda de una solución integral de las mismas.

c) el reduccionismo jurídico, que limita la evaluación de las situaciones a una sola perspectiva: el derecho, el cual tiene como su autoridad natural al juez (24).

\section{Críticas al método deliberativo}

A esta propuesta se le han hecho las siguientes críticas: en primer lugar, en la etapa de deliberación sobre los hechos, de acuerdo con Tomás Domingo, esta propuesta podría enriquecerse si en ella se "tuviera más en cuenta el papel de la imaginación y de la narración” (26, p.76). Se resaltan dos dimensiones del proceso deliberativo: la argumentación y la interpretación, en donde, la segunda es una pieza esencial: "interpretar las acciones, las personas, sus conflictos, etc. No se trata solo de describir, ni tampoco de explicar, sino básicamente de comprender" (26, p.70).

En segundo lugar, en la etapa de deliberación sobre los valores no se encuentra una definición, clasificación y propuesta de ponderación de estos en caso de conflicto, lo que podría dificultar su aplicación en la práctica. Esta clarificación sería importante, más aún cuando se está inmerso en una sociedad pluralista en la que los valores pueden ser tantos cuantos conceptos de mundo existan. Por otra parte, en caso de que la naturaleza de los problemas morales no estuviera expresada en el lenguaje de los valores sino en principios morales, deberes, obligaciones, normas o creencias, ¿cómo estos podrían ser resueltos a través de esta metodología?

\section{Discusión}

El método propuesto por Gracia, fundamentado en el constructivismo axiológico y la deliberación, parte del reconocimiento del ser humano como un ser moral, un ser capaz de tomar decisiones en favor de su autorrealización. Se hace a través de la aprehensión de la realidad de manera dual, intelectual y emocional, capacidad que ha sido denominada por Xavier Zubiri como la inteligencia sentient"(11) y por los pescadores del Caribe colombiano como el ser humano sentipensante( ), dimensiones humanas indisolubles, interdependientes y esenciales, cuya importancia recientemente ha sido reconocida en occidente donde, por mucho tiempo, se dio un reconocimiento único o preponderante a la dimensión intelectual en el ámbito científico y social.

El progreso de la neuroética y de otras ciencias de la mente, en las últimas décadas, ha permitido avanzar en el conocimiento del desarrollo del cerebro y de su estructura funcional, a partir del cual se ha podido fundamentar estas nociones de inteligencia sentiente o de ser humano sentipensante. A este respecto, las investigaciones realizadas en este campo, a pesar de lo reciente y parciales de las mismas, permiten llegar a algunas con- 
clusiones que vienen a soportar lo anteriormente expuesto:

a) el redefinir a los seres humanos como criaturas neuro-culturales, es decir, el fruto de la interacción dinámica entre el individuo como un organismo vivo, producto de una herencia genética y biológica, y su medio ambiente, social y cultu$\operatorname{ral}(27)$;

b) el comenzar a comprender el papel fundamental que juegan los instintos, las emociones y los pensamientos en la conducta moral(28);

c) y el identificar una estructura moral previa a nivel cerebral, a partir de la cual el ser humano construye su moral, como muchos estudiosos previamente lo han propuesto (29, $30)$.

La deliberación ha sido reconocida como una habilidad innata del ser humano necesaria para su supervivencia y desarrollo, que le permite tomar decisiones racionales y prudentes en condiciones de incertidumbre y contingencia. Es de reconocida importancia y vigencia en campos especializados del saber como la medicina, el derecho, la política y la ética. Específicamente, en las ciencias de la salud, es la base para el análisis de los casos clínicos y la toma de decisiones; de ahí la importancia de la cualificación de esta habilidad en los profesionales de la salud a través de las instituciones de educación y los comités de bioética, para su ejercicio profesional y la vida. Respecto a esto, Gracia anota: "Deliberar es una práctica, una habilidad que se aprende con el ejercicio. Y sobre todo, es una actitud, un estilo de vida, que debe- ría aprenderse y ejercitarse desde la niñez" (19, p. 70).

El método de toma de decisiones en bioética clínica aquí propuesto viene a reivindicar la deliberación en el contexto clínico y la importancia del estudio a profundidad del caso clínico. De manera general, su método podría identificarse con el casuismo, definido como la realización de "juicios probables en situaciones concretas e individuales, a la vista de todas las circunstancias y en el contexto donde se produce el caso, teniendo en cuenta los diferentes puntos de vista y los intereses de todos los afectados" (2, p. 181). En él, el modelo de razonamiento de manera característica se basa en la prudencia, virtud que Aristóteles describe como la habilidad del ser humano para "un modo de ser racional, verdadero y práctico, respecto a lo que es bueno para el hombre" (31, p. 167); y en el conocimiento práctico, que se caracteriza por ser: "concreto, porque su verdad se apoya en la experiencia directa; temporal, porque las verdades basadas en la experiencia práctica no son necesariamente universales; y probable, porque la conclusión que se aplica a la generalidad de los casos, no se aplica necesariamente a todos los casos" (2, p. 169).

Los aportes más importantes de esta propuesta son: la valoración de la historia clínica como el elemento básico para el estudio y el análisis del caso clínico; el reconocimiento de la deliberación prudente como el eje fundamental para la valoración de las situaciones problemas; el análisis que se hace de las dificultades intrínsecas y extrínsecas 
que pueden dificultar el proceso deliberativo, las cuales deben ser reconocidas de manera oportuna, a fin de evitar que se constituyan en un obstáculo en esta tarea; y la metodología propuesta es clara y sencilla para la identificación y elección de los problemas morales a estudio y de los cursos de acción para su resolución.

Por otra parte, algunas debilidades en el método propuesto a tener en cuenta para su cualificación son las siguientes:

a) En relación con los valores morales, no se evidencia una clara definición y clasificación de los mismos, así como una propuesta de ponderación de estos en caso de conflicto, lo que podría dificultar su aplicación. De igual forma, no se establece una relación entre estos y otros aspectos a considerar en el análisis, como los principios morales, deberes, obligaciones, normas o creencias;

b) $\mathrm{Al}$ centrar el estudio en el caso particular se desconoce lo universal, lo cual es también algo necesario e irrenunciable para el estudio integral del caso clínico. Por esta razón, la calidad de sus recomendaciones depen-

\section{Referencias}

1. Sánchez M. Bioética en Ciencias de la Salud. Madrid: Elsevier Masson; 2013. $536 \mathrm{p}$.

38 2. Ferrer J., Álvarez J. Para fundamentar la bioética. Teorías y paradigmas teóricos de, en últimas, de la calidad de los miembros participantes y el proceso deliberativo que desarrollen.

Finalmente, es de reconocer la obra de Gracia como uno de los aportes más importantes realizados al estudio de los métodos de toma de decisiones en bioética clínica en lengua española, la cual se constituye como una herramienta útil en la construcción y enriquecimiento del conocimiento en este campo para los profesionales de la salud y los comités bioéticos clínicos.

\section{Agradecimientos}

Al Doctor Fabio Alberto Garzón, Doctor en Filosofía, Máster en Bioética Clínica y Fundamental y Docente Investigador del Doctorado en Bioética y de la Facultad de Educación y Humanidades de la Universidad Militar Nueva Granada, por su orientación en la elaboración del presente escrito.

\section{Conflicto de intereses}

Los autores declaran no tener conflictos de intereses. en la bioética contemporánea. Bilbao: Editorial Universidad Pontificia Comillas; 2003. $559 \mathrm{p}$.

3. Faúndez J. La bioética de Diego Gracia. $1^{\text {a }}$ ed. Madrid: Editorial Triacastela; 2013. 
4. Colombia, Colciencias. Documento Guía. Servicio permanente de indexación de revistas de ciencia, tecnología e innovación colombianas. Bogotá: Colciencias; 2010. [Citado: 2018 mayo 10]. Disponible en: http://www.colciencias. gov.co/sites/default/files/upload/paginas/ M304PR02G01-guiaserviciopermanente-indexacion.pdf

5. Potter V. Bioética puente, bioética global, bioética profunda. Cuadernos del Programa Regional de Bioética, OPSOMS. 1997;7:21-35.

6. Gracia D. Bioética clínica. Bogotá: Editorial El Búho Ltda.; 1998.

7. Ferrer J. Historia y fundamento de los comités de ética. En: Martínez J., ed.. Comités de Bioética. Madrid: Editorial Universidad Comillas; 2003. p. 17-42.

8. Green J, Thorogood N. Qualitative methods for health research. 3a ed. Los Angeles: SAGE Publications Ltd.; 2014.

9. Flick U. Introducción a la investigación cualitativa. 3a ed. Madrid: Ediciones Morata, S.L.; 2012. 322 p.

10. Tezanos A. Una etnografía de la etnografía. Aproximaciones metodológicas para la enseñanza del enfoque cualitativo-interpretativo para la investigación social. Bogotá: Antropos.; 1998. 172 p.

11. Zubiri X. Inteligencia sentiente. 2a ed. Madrid: Alianza Editorial - Sociedad de Estudios y Publicaciones; 1981.

12. Real Academia Española. Diccionario de la Lengua Española [Internet]. Madrid: Real Academia Española; 2014. [Citado: 2015 Mayo 10]. Disponible en: http://www. rae.es

13. Gracia D. La deliberación moral. Boletín de la Academia Chilena de Medicina. 2001;38:29-45.

14. Gracia D. Teoría y práctica de la deliberación moral. En: Feito L, Gracia D,
Sánchez M, editores. Bioética: el estado de la cuestión. Madrid: Editorial Triacastela; 2011. p. 101-154.

15. Gracia D. Philosophy: Ancient and contemporary approaches. In: Sugarman $\mathrm{J}$, Sulmasy D, editors. Methods in medical ethics. 2a ed. Washington, D.C.: Georgetown University Press; 2010. p. 55-71.

16. Gracia D. Deliberation and consensus. In: Chadwick R, Have T, Meslin E, editors. The SAGE Handbook of Health Care Ethics. 2a ed. Londres: SAGE Publications; 2011. p. 84-94.

17. Gracia D. La deliberación moral. El papel de las metodologías en ética clínica. En: Sarabia J, De los Reyes M, eds. Comités de ética asistencial. Madrid: Asociación de Bioética Fundamental y Clínica; 2000. p. 21-41.

18. Gracia D. La deliberación moral: el método de la ética clínica. En: Gracia D., Júdez J., eds. Ética en la práctica clínica. Madrid: Editorial Triacastela; 2004. p. 21- 35.

19. Gracia D. Teoría y práctica de los comités de ética. En: Martínez J, ed. Comités de bioética. España: Universidad Pontificia Comillas; 2003. p. 59-70.

20. Gracia D. Fundamentación y enseñanza de la bioética. 2a ed. Bogotá: Editorial El Búho Ltda.; 2000.

21. Gracia D. Valor y precio. Madrid: Editorial Triacastela; 2013.

22. Gracia D. El estatuto epistemológico de la bioética. En: Feito L, Domingo T, editores. Investigación en Bioética. Madrid: Universidad Rey Juan Carlos - Dykinson, S.L.; 2009. p. 57-81

23. Gracia D. Fundamentos de Bioética. 3a ed. Madrid: Editorial Triacastela; 2008.

24. Gracia D., Pose C. Introducción a la bioética: origen, fundamentación y méto- 
do. En: Gracia D., editor. La Bioética en la educación secundaria. Madrid: Ministerio de Educación y Ciencia; 2007. p. 9-50.

25. Gracia D. Procedimientos de decisión en ética clínica. 3a ed. Madrid: Editorial Triacastela; 2008.

26. Domingo T. Bioética en perspectiva hermenéutica: hacia un método hermenéutico deliberativo. En: Feito L, Gracia $D$, Sánchez M, editores. Bioética: el estado de la cuestión. Madrid: Editorial Triacastela; 2011. p. 57-77.

27. Evers K. Neuroethics. En: Runehov A., Lluis O., eds. Encyclopedia of Scien- ces and Religions. Nueva York: Springer; 2013. p. 1466-71.

28. Suhler C., Churchland P. The neurobiological basis of morality. En: Illes J., Sahakian B., eds. The Oxford Handbook of Neuroethics. Nueva York: Oxford University Press; 2011. p. 33-58.

29. Cortina A. Neuroética y neuropolítica. Madrid: Tecnos S.A.; 2010.

30. Damasio A. Y el cerebro creó al hombre. España: Destino S.A.; 2010.

31. Aristóteles. Ética a Nicómaco. Madrid: Gredos S.A.; 2014 Trad. 